\title{
MR-safe multisegmental vibration device for cortical mapping of paraspinal afferent input
}

This paper was downloaded from TechRxiv (https://www.techrxiv.org).

\section{LICENSE}

CC BY 4.0

SUBMISSION DATE / POSTED DATE

$11-12-2021 / 16-12-2021$

\section{CITATION}

Schibli, Louis; Gandia, Robert; Buck, Roger; Staempfli, Philipp; Meier, Michael; Schuetz, Philipp (2021): MRsafe multisegmental vibration device for cortical mapping of paraspinal afferent input. TechRxiv. Preprint. https://doi.org/10.36227/techrxiv.17161883.v1

$\mathrm{DOI}$

10.36227/techrxiv.17161883.v1 


\title{
MR-safe multisegmental vibration device for cortical mapping of paraspinal afferent input
}

\author{
Louis Schibli a, Robert Gandia a, Roger Buck a, \\ Philipp Staempfli ${ }^{\mathrm{c}}$, Michael Meier ${ }^{\mathrm{b}, \mathrm{c}}$, Philipp Schuetz ${ }^{\mathrm{a}, *}$ \\ ${ }^{a}$ Lucerne Unviersity of Applied Sciences and Arts, Horw, Switzerland \\ ${ }^{b}$ Balgrist University Hospital, Department of Chiropractic Medicine, Integrative Spinal Research, Zurich, Switzerland \\ ${ }^{c}$ University of Zurich, Zurich, Switzerland \\ ${ }^{c}$ Department of Psychiatry, Psychotherapy and Psychosomatics, Hospital of Psychiatry, University of Zurich, Zurich, Switzerland
}

\begin{abstract}
Lack of knowledge about the cortical organization of paraspinal afferent input in humans' limits investigations of the role of neurobiological mechanisms in low back pain pathophysiology. Suitable MR-compatible stimulation methods that can produce detailed cortical maps of thoracolumbar afferent input are lacking. Therefore, the objective of this study was to design an MR-safe stimulation device that can apply randomized mechanical (vibrotactile) stimulation to different thoracolumbar segments at predefined amplitudes. The system developed relies on vibration units fixed to the study subject, a pneumatic system that enables the adjustment of the vibration amplitude at different frequencies and a control system that enables the interaction with the operator and synchronization to the MR-system. The presented pneumatic vibrotactile stimulation device (pneuVID) allows vibrotactile stimulation between $5 \mathrm{~Hz}$ and $120 \mathrm{~Hz}$ at different paraspinal locations. Two vibration frequencies $(20 \mathrm{~Hz} / 80 \mathrm{~Hz})$ for differential paraspinal mechanoreceptor stimulation were evaluated. The stimulation at $20 \mathrm{~Hz}$ and $80 \mathrm{~Hz}$ is highly reproducible and consistent, both during two consecutive MRI measurements as well as during one year of operation under study conditions. PneuVID also enables the adjustment of the vibration amplitude for both frequencies to correct for the inverse-frequency dependency of the amplitude. To conclude, the developed system enables a controlled vibrotactile stimulation during (functional) MRI-measurements and thereby constitutes a promising tool for generating detailed cortical maps of paraspinal afferent input.
\end{abstract}

\section{Keywords: vibration, magnetic resonance imaging, muscle spindle, proprioception, low back pain}

\section{Introduction}

Persistent pain such as chronic low back pain (LBP, the leading cause of years lost to disability worldwide ${ }^{1}$ ) can alter brain-body maps which might have pathological significance ${ }^{2}$. Non-invasive human brain imaging techniques such as functional magnetic resonance imaging (fMRI) with its high spatial resolution in combination with vibrotactile stimulation provide suitable tools for the investigation of the cortical topography of various sensory inputs of different body parts ${ }^{3}$. However, very little is known about a potential cortical topographic organization of paraspinal afferent input of the human back (e.g., along the thoracolumbar axis). Detailed cortical maps of paraspinal afferent input might be crucial to further explore their pathological meaning in persistent LBP, yet suitable stimulation methods are lacking. Although MR-compatible vibration devices exist ${ }^{4,5}$, none of them is capable of applying controlled vibrotactile stimulation at different thoracolumbar segments with appropriate amplitude in supine subjects. Vibration frequencies around $80 \mathrm{~Hz}$ (and amplitudes between $0.5-1 \mathrm{~mm}$ ) have been shown to modulate the activity profile of muscle spindles and therefore proprioceptive information ${ }^{6}$. In contrast, vibration frequencies around $20 \mathrm{~Hz}$ mainly target superficial mechanoreceptors (i.e. Meissner corpuscles ${ }^{7}$ ). Therefore, applying different vibration frequencies to the human back in combination with fMRI might reveal the cortical targets and organisation of paraspinal proprioceptive and tactile afferent inputs. The objective of this study was to develop an MR-safe stimulation device (pneumatic vibration device, pneuVID) that can apply vibrotactile stimulation to different thoracolumbar segments and to characterize stimulation parameters such as the amplitude and its stability for two relevant frequencies $(20 \mathrm{~Hz} / 80 \mathrm{~Hz})$. This is the first apparatus specifically designed for paraspinal tissue vibration on different segmental levels in an MR environment. 


\section{Materials and methods}

\subsection{System layout}

PneuVID provides vibrotactile stimulation to the human back with adjustable parameters during MR imaging. These parameters are the stimulation frequency, the duration of the stimulation, the inter-stimulation time, the amplitude, and the stimulation position. In the following section, an overview of the overall operation of the system is provided. Details on the individual components are presented in subsequent sections. The system can be divided into three subsystems (Figure 1):

The control module (see section 2.5) enables the operator to setup the experiment, i.e., defining the parameters of the experimental run. The system operates one pair of vibration units (VU) at the same time, which allows bilateral stimulation at different segmental levels. The operator can set the duration of the stimulation, the inter-stimulation time, the frequency (between $5-150 \mathrm{~Hz}$ ) and the amplitude of the stimulation. All parameters except the amplitude can be set arbitrarily. The control module consists of a Raspberry Pi 3B+ connected to a screen. Together with the air compressor, the control module is located in the control room. The control module is connected to the valve box (see section 2.4), the second part of the system, via a fibre optic cable. The valve box is a metallic enclosure acting as a Faraday cage and is attached to the end of the MR table inside the instrument room. The valve box contains a second Raspberry Pi 3B+, which is used to control the 12 fast-switching valves installed in the box. The step signals of the Raspberry Pi are raised to the required voltage level for switching the valves by means of a level shifter. To enable the communication between the two Raspberry Pi's via the fibre optic cable, each of them is connected to a media converter (see section 2.4). Compressed air is supplied via tubes at $1.5 \mathrm{bar}$ to the valves. Pulses of compressed air are generated by fast-switching valves (see section 2.4.1) in the valve box and are directed through tubes to the VU (see section 2.2). Each tube has two VUs attached to it connected via a T-junction and a total of 20 VUs are attached to the system. The VUs are attached to the subject's back (see section 2,3), and the subject lies on the VUs on a special pillow during the MRI scan.

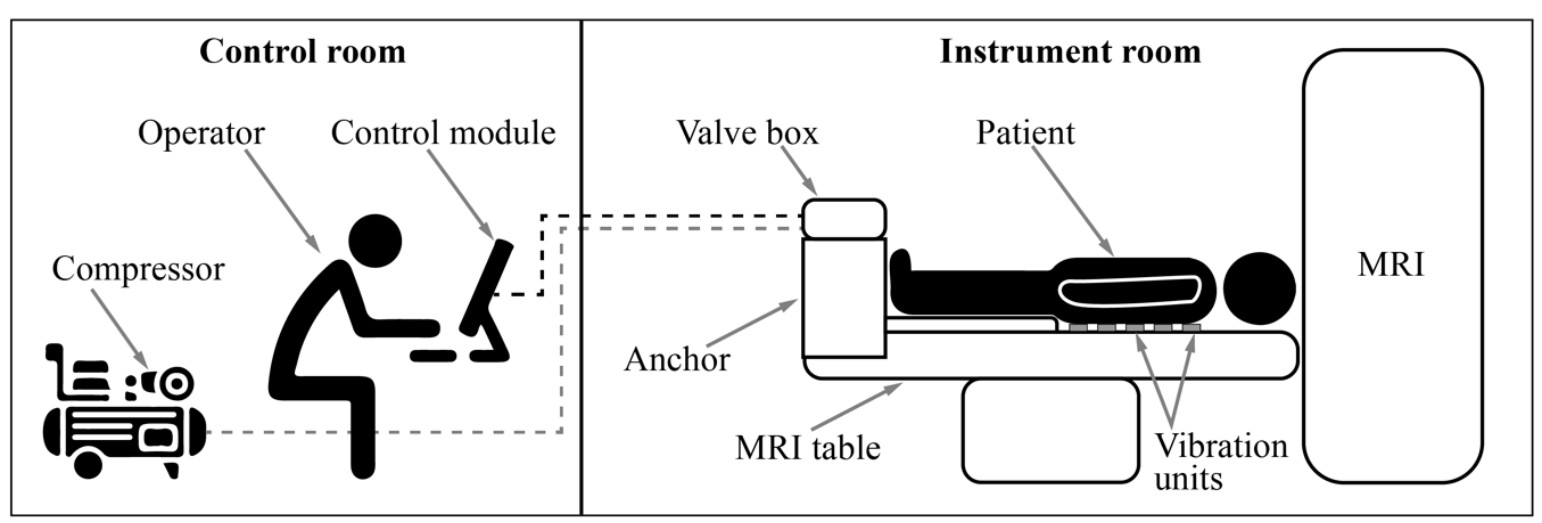

Figure 1: System layout and positioning of the different parts in the MR environment. The control room is shielded from the electromagnetic fields of the MRI. The connections of the Raspberry $\mathrm{Pi} 3 \mathrm{~B}+$ and the compressor to the valve box are established via a dedicated and shielded junction of the two rooms.

\section{2. $V U$ design and fabrication}

The VU (Figure 2, 50x30x7mm) was fabricated manually. For the casting process, molds were used which were printed in PLA by a 3D printer (i3 MK3S, Prusa, Czech Republic). The VU was built up from top (1 $1^{\text {st }}$ layer) to bottom (scaffold) and was constructed in three layers of RTV2 silicone (SILIXON, Silikonfabrik, Deutschland). The $1^{\text {st }}$ layer defines the shape of the oscillating $2^{\text {nd }}$ layer and has direct contact with the study subject. The $1^{\text {st }}$ layer was cast from a RTV2 silicone (SILIXON40, Silikonfabrik, Deutschland) with a target shore-hardness of 40ShA. After each layer, the silicone was heated on a hot plate at $50^{\circ} \mathrm{C}$ for 5 minutes to allow the two components of the silicone to crosslink quickly. Thereafter, the layer exhibited sufficient stability to prevent mixing with other layers. The $2^{\text {nd }}$ layer was made of a RTV2 silicone (SILIXON10, Silikonfabrik, Deutschland) with a target shorehardness of 10ShA. During stimulation, this layer is stretched up to $9 \mathrm{~mm}$ (see section 3.1) by pulses of compressed 
air. A pigment was added to this layer to improve reflectivity for the laser distance sensor (see section 2.6) and to prevent this layer from being transparent. The $3^{\text {rd }}$ layer consists of the same silicone type as the first layer. This layer serves as a host for the scaffold and allows easy positioning of the VU. The scaffold was 3D printed in PLA (i3 MK3S, Prusa, Czech Republic) and was embedded in the $3^{\text {rd }}$ layer. The deflection plate was inserted between the $2^{\text {nd }}$ and $3^{\text {rd }}$ layer. This structuring element was printed in PLA and ensures that the compressed air pulse expands predominantly the $2^{\text {nd }}$ layer (lamella). The silicone tube (outer diameter $2 \mathrm{~mm}$, inner diameter $1 \mathrm{~mm}$, silicone tube without inserts 1.0x1.0, Maagtechnic, Switzerland) was positioned under the $2^{\text {nd }}$ layer and then coated with RTV2 silicone (SILIXON40). The mixing ratios of the individual layers are listed in Table 1.

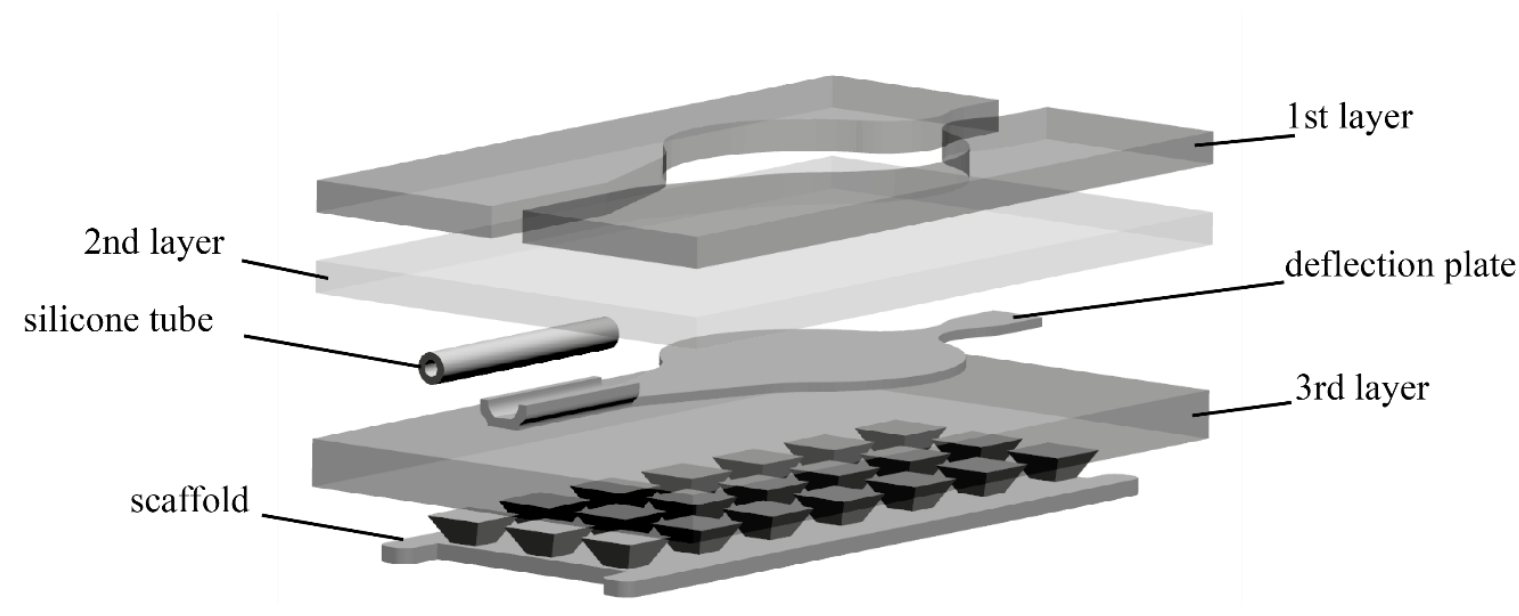

Figure 2: Visualisation of the triple-layer VU. The scaffold and the deflection plate were made of PLA whereas the three layers and the tube were made of different RTV2 silicones.

Table 1: Mixture ratio of base to curative of the RTV2 silicone of the different silicone layers.

\begin{tabular}{|l|l|l|l|l|}
\hline Layer & Total mass & Base & Curative & Base : Curative \\
\hline No. 1 & $2.8 \mathrm{~g}$ & $4 / 3 \mathrm{~g}$ & $22 / 15 \mathrm{~g}$ & $1.1: 1$ \\
\hline No. 2 & $3.5 \mathrm{~g}$ & $5 / 3 \mathrm{~g}$ & $11 / 6 \mathrm{~g}$ & $1.1: 1$ \\
\hline No. 3 & $4.5 \mathrm{~g}$ & $15 / 7 \mathrm{~g}$ & $33 / 14 \mathrm{~g}$ & $1.1: 1$ \\
\hline
\end{tabular}




\subsection{Fixation of the VUs on study subjects}

Attachment of the VU to the subject (Figure 3) is performed in the instrument room while the subject is seated on the MR scanner table. Beforehand, the positions of the VU on the study subject's back is determined by palpation of the spinous processes and marked with a skin-compatible coloured pencil by an experienced physiotherapist. The VUs are attached to the back using a sugar-based adhesive which is skin-friendly, easy to remove, and yet allows short-term fixation of the difficult-to-bond VU with silicone surface.

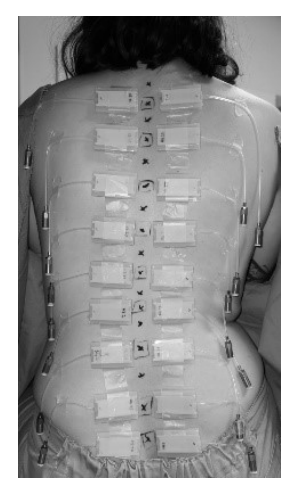

Figure 3: Attachement of the VUs on the back of a study subject.

\subsection{Valve box}

The valve box is positioned in the instrument room by means of an anchor at the foot of the MR table (see Figure 1). The box was designed as a Faraday cage $(262 \times 182 \times 90 \mathrm{~mm})$ (RND components, Switzerland) and includes the following key components:

I Raspberry Pi 3B+ (Raspberry Pi Foundation, England)

ๆ Media converter MC220L V4 (TP-Link, Shenzhen, China)

ๆ Level shifter (own production)

I Battery, PB-20 PD, 20'000mAh, 2x USB output 5 V, 2.1 A (RealPower, Germany)

ๆ Battery, Gens Ace, 4'000mAh, 11.5 V, 25C (Grepow, Shenzhen, China)

ๆ $12 \mathrm{x}$ fast-switching valves MHE2-MS1H-3/2G-QS-4-K (Festo, Germany)

I 2x throttle check valves GR-QS-4 (Festo, Germany)

The valve box has two openings for incoming lines and two openings for outgoing lines: The inputs are used for the tubing from the compressor ( $8 \mathrm{~mm}$ outer diameter, $6 \mathrm{~mm}$ inner diameter) and the fibre optic cable from the control module. From each of the outlets, 5 tubes $(6 \mathrm{~mm}$ outer diameter, $4 \mathrm{~mm}$ inner diameter) are led to the VUs. The four openings are each provided with an aluminum tube, which serves as a filter for the MR system's RF signals and prevents the system's RF signal from interacting with the MR system. Other components and connections installed in the box are described in the following sections $(2.4 .1-2.5)$. 


\subsubsection{Pneumatic setup}

The pneumatic setup of the system (Figure 4) consists of 12 fast-switching 3/2-way valves (MHE2-MS1H-3/2GGS-4-K, Festo, Germany), two throttle check valves (AS2002F-04, SMC, Germany), two manifolds (KM11-0408-10, SMC, Germany), 10 check valves (AKH04-00, SMC, Germany) and the associated connectors and tubes. Two of the fast-switching valves are used as OR valves (inlet or outlet valve). These inlet or outlet valves supply the compressed air either through the regulated or the unregulated path. The remaining 10 fast-switching valves generate the compressed air pulses that causes the VU to expand (oscillating valves). Two VUs are connected to each of the oscillating valves and only one of the valves is triggered at a time. The oscillating valves work as a 3/2-way valve, i.e., due to the rectangle signal type the valve was $50 \%$ of the stimulation time connecting [In] to [Out 1] and $50 \%$ of the time [Out 1] to [Out 2]. During the connection from [In] to [Out 1], the VU inflates and expands. As soon as [Out 1] is connected to [Out 2], the VU is deflated, and compressed air flowed from the VU to the outlet valve. This escape route prevents the VU from permanently inflating and the individual pulses are well separated.

For the study setting, the system was designed to operate two different frequencies $(80 \mathrm{~Hz} / 20 \mathrm{~Hz})$ of the oscillating valves with a constant amplitude of the VU. With a lower frequency, the time for the expansion and deflation of the VU is longer, resulting in a larger amplitude of the VU. To equalise the amplitudes at both frequencies, the volume flow of the lower frequency had to be regulated by means of two throttle valves. The amplitude at each frequency can be adjusted to the desired value by these throttle valves. It is important to note that the amplitude with regulation at the higher frequency cannot exceed the amplitude reached without regulation at the same frequency (Figure 7). Frequencies from $5-150 \mathrm{~Hz}$ can be operated by the system. If the inlet valve is set to regulate the compressed air, the outlet valve is also regulating the exhaust air. The check valves attached to the [Out 2] of each oscillating valve prevent the compressed air from flowing back to the VU. They have a minimum activation pressure of 5Pa. All connections inside the box were realised using tubes $(4 \mathrm{~mm}$ outer diameter and $2 \mathrm{~mm}$ inner diameter) and T-junctions.

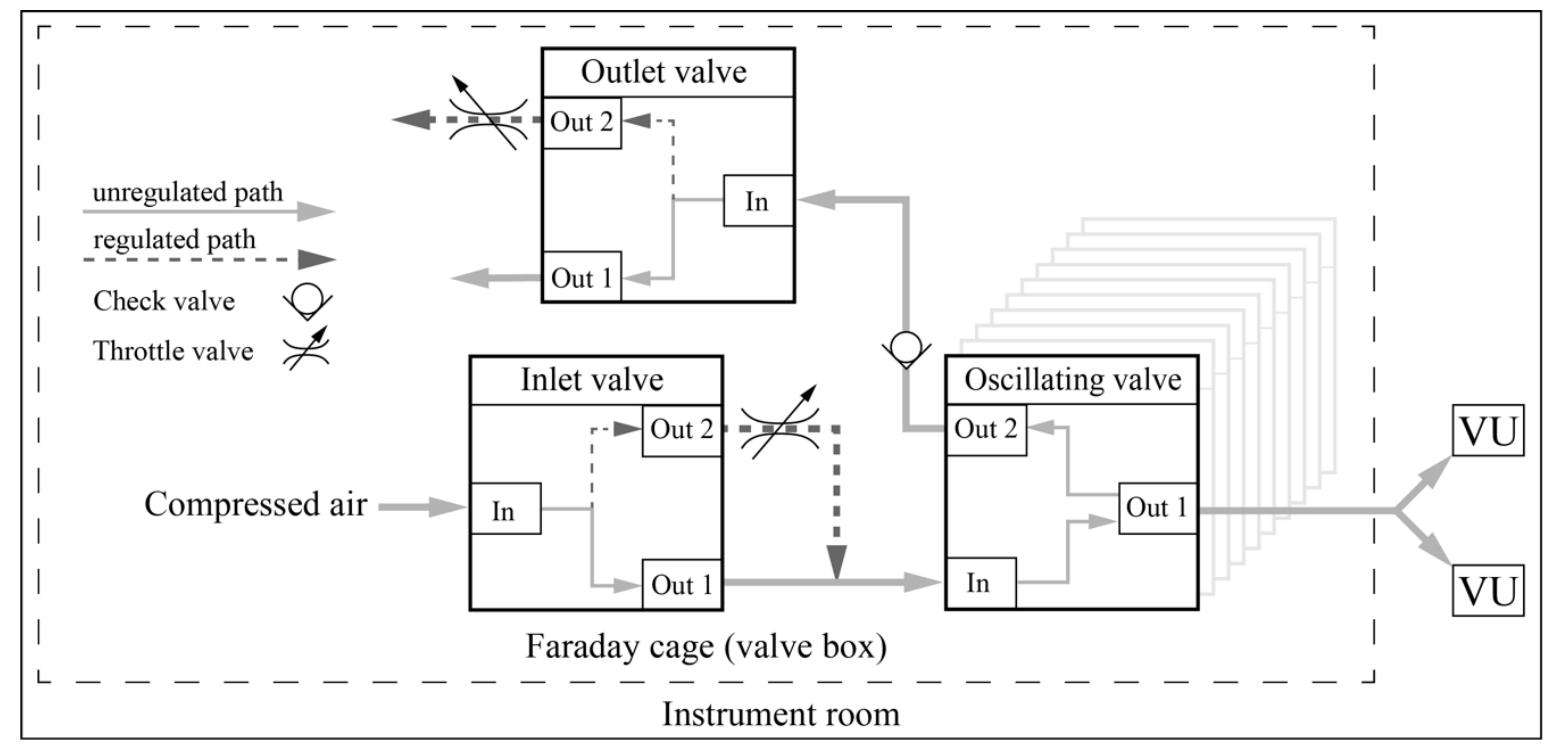

Figure 4: Schematic representation of the pneumatic structure of the valve box. Both the regulated and the unregulated paths are shown, whereas only one is active at a time. A total of 12 fast-switching valves are installed. 


\subsubsection{Electronic setup}

The fast-switching valves require an electric pulse of $24 \mathrm{~V}$ and up to $1 \mathrm{~A}$ peak current. This pulse could not be generated with the Raspberry Pi 3B+ microcontroller. Therefore, the general-purpose input/output port (GPIO) of the Raspberry Pi is connected to motor drivers (ULN2803A, Texas Instruments, Dallas, USA). Upon activation, this motor driver supplies the required electric signal to the fast-switching valve. The motor drivers are powered by a Lithium-Polymer powerpack (Gens Ace 4'000, Grepow, Shenzhen, China) with a capacity ratio of 25C to ensure a steady supply of the motor driver. Each motor driver switches up to 8 valves. A printed circuit board has been designed inhouse to host 2 motor drivers to control the 10 oscillating valves. The inlet and outlet valves are both powered by the lithium-ion battery and are connected to the circuit board. As these valves have a higher electrical power demand, they are each controlled by a separate MOSFET (IRLD024 \& IRFD9120, Vishay Siliconix, Malvern, USA) to prevent the motor drivers from overheating.

\subsubsection{Control software}

The control software was implemented in python3. During the initialisation, the valve box sets up a connection channel to the control module (see section 2.1) via network TCP/IP communication, exchanges text messages and enters the "ready-to-measure" stage. The valve box interprets three distinct types of messages:

A) Stop signal caused the valve box to stop the excitation schedule after the completion of the current pulse

B) Measurement program: The measurement program consisting of a sequence of VU ID, excitation frequency, stimulation duration and inter-stimulation time and is transmitted as a telegram

C) Measurement start signal: A start message for the program

Upon receipt of the measurement program, the valve box changes to the "measurement-ready" state. After a TTL signal is received from the MR system upon the start of the MR measurement sequence (see section 2.5 for details), the valve box executes the parsed telegram of the excitation program. For each sequence element, the valve box activates a PWM signal (generated by the GPIO library) on the GPIO-Pin connected to the oscillating valve with the requested stimulation frequency and (randomized) duration and then stops the PWM signal again. Subsequently, the valve box checks whether a stop signal is received. If no stop signal is received, the process above will be repeated with the next sequence element. In case a stop signal is received, the valve box stops the parsing of the excitation program, sends a stop confirmation signal to the control box, and returns to the "readyto-measure" state. 


\subsection{Control box and software}

The control box enables the communication with the operator, the setup of the stimulation parameters (number of vibration events, vibration frequency, stimulation duration and inter-stimulation time), manages the communication with the valve box (see section 2.4) and enables the synchronization with the MR system. The control box consists of a Raspberry Pi 3B + microcontroller with an external 7" Raspberry Pi display. The control box and the valve box are connected via a TCP/IP network-connection established through two media converters (MC220L V4, TP-Link, China) connected via a fibre optical cable. Each media box is connected to the Raspberry Pi microcontroller via a conventional gigabit ethernet connection. The fibre optical connection was chosen to reduce the impact of electromagnetic signals as well as preventing additional conducting structures to the MRmeasurement room. On the control box, one of the general-purpose input channels is used to collect the TTLsignal of the MR-device issued on the start of the MR measurement sequence. As the TTL-signal has a level of $5 \mathrm{~V}$ and the Raspberry Pi microcontroller can only handle $3.3 \mathrm{~V}$, the signal level is reduced by a passive voltage splitting circuit based on a 3.3 and $1.1 \mathrm{kohm}$ resistor.

On the display, the operator can choose the number of stimulations applied, two different stimulation frequencies, the duration of the stimulation, the inter-stimulation time (range) and the VUs to be activated. As soon as the required information is entered the control box changes to the "ready-to-measure" state. Upon the start of the measurement, the control box changes to the "measurement" status and the software generates a random sequence of requested number of stimulations with the chosen frequencies and random stimulation durations in the operatorselected range. In a last step, the sequence of stimulations is pseudorandomized (no more than 3 consecutive stimulations at the same VU, no more than two consecutive equal frequencies at the same VU). After the generation of the stimulation sequence consisting of VU ID, vibration frequency, stimulation duration and inter-stimulation time, the system is in a trigger wait state for subsequent synchronization with MR data acquisition. In the "measurement" mode, the control software only accepted the "cancel" button press, which sends a stop signal to the valve box and sets the control box into the "ready-to-measure" mode. As soon as the valve box returns a measurement completed signal, the control box returns to the "ready-for-measurement" state.

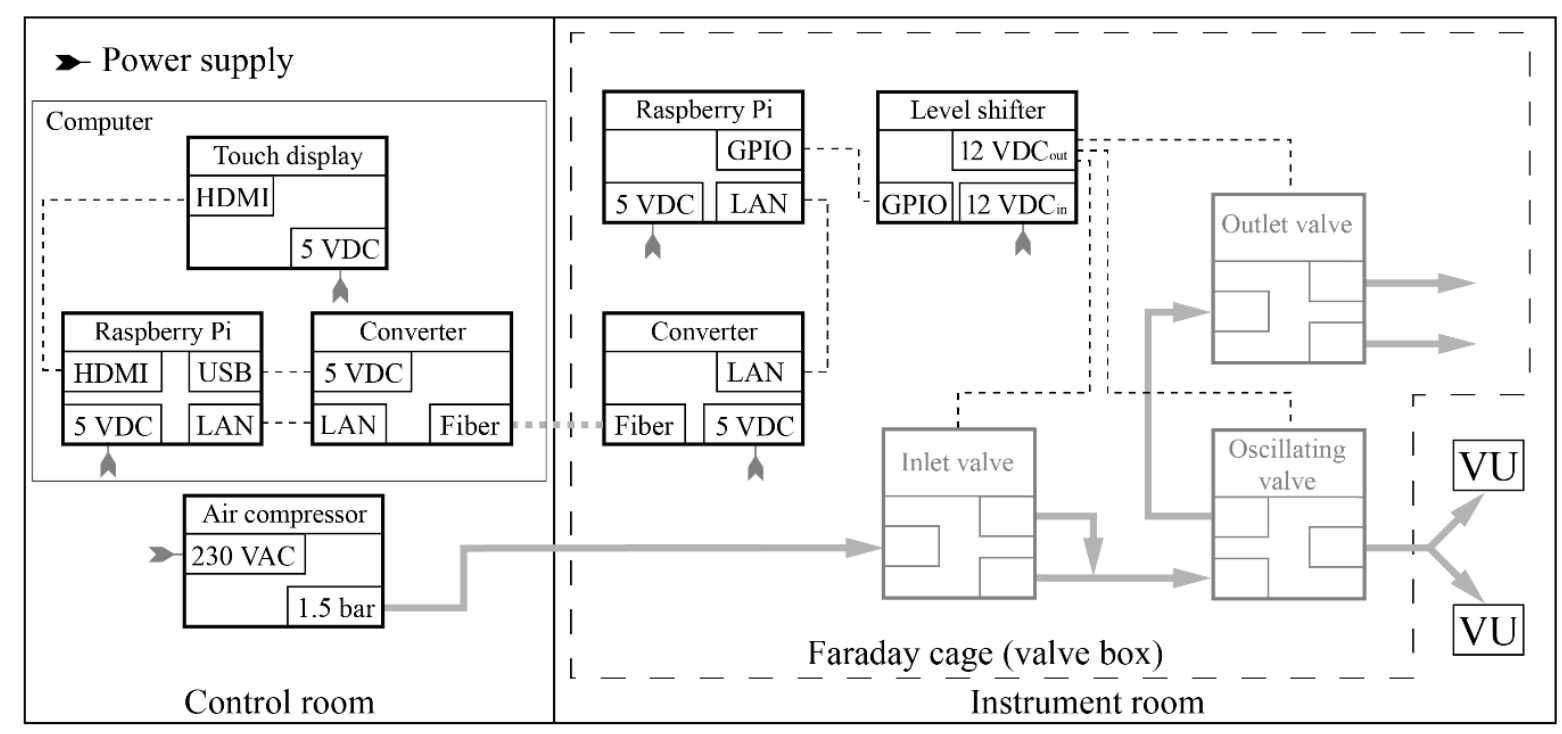

Figure 5: Schematic representation of the electronic interconnection between the individual components. For simplification, only one oscillating fast-switching valve is shown; in the valve box, 10 oscillating valves are connected to the level shifter. 


\subsection{Measurement setup for the assessment of the VU amplitude}

A triangulation laser (AWLG 008 s, Welotec, Germany) was used to measure the extension of the VU's lamella and enabled thereby the quantification of the stimulation amplitude. The triangulation laser can be operated at a sampling rate of up to $5000 \mathrm{~Hz}$ and an accuracy of $\pm 0.5 \mathrm{~mm}$. The triangulation laser was mounted perpendicularly over the centre of the VU's lamella. The laser beam was focused on the surface of the second layer (lamella) of the VU and measured its deflection. One VU was measured at a time, whereby two VU had to be connected to the system. The output signal (analogue voltage output) was recorded with a digital oscilloscope (OpenScope MZ, Digilent, USA) using the open-source software Waveformslive (Digilent, USA). The recorded data was transferred to the computer via the USB interface, which served as the oscilloscope's power supply. A conversion coefficient was set for the triangulation laser to ensure that the output voltage corresponds to the measured distance in millimetres. The coefficient was determined using gauge blocks (tolerance class 0 ).

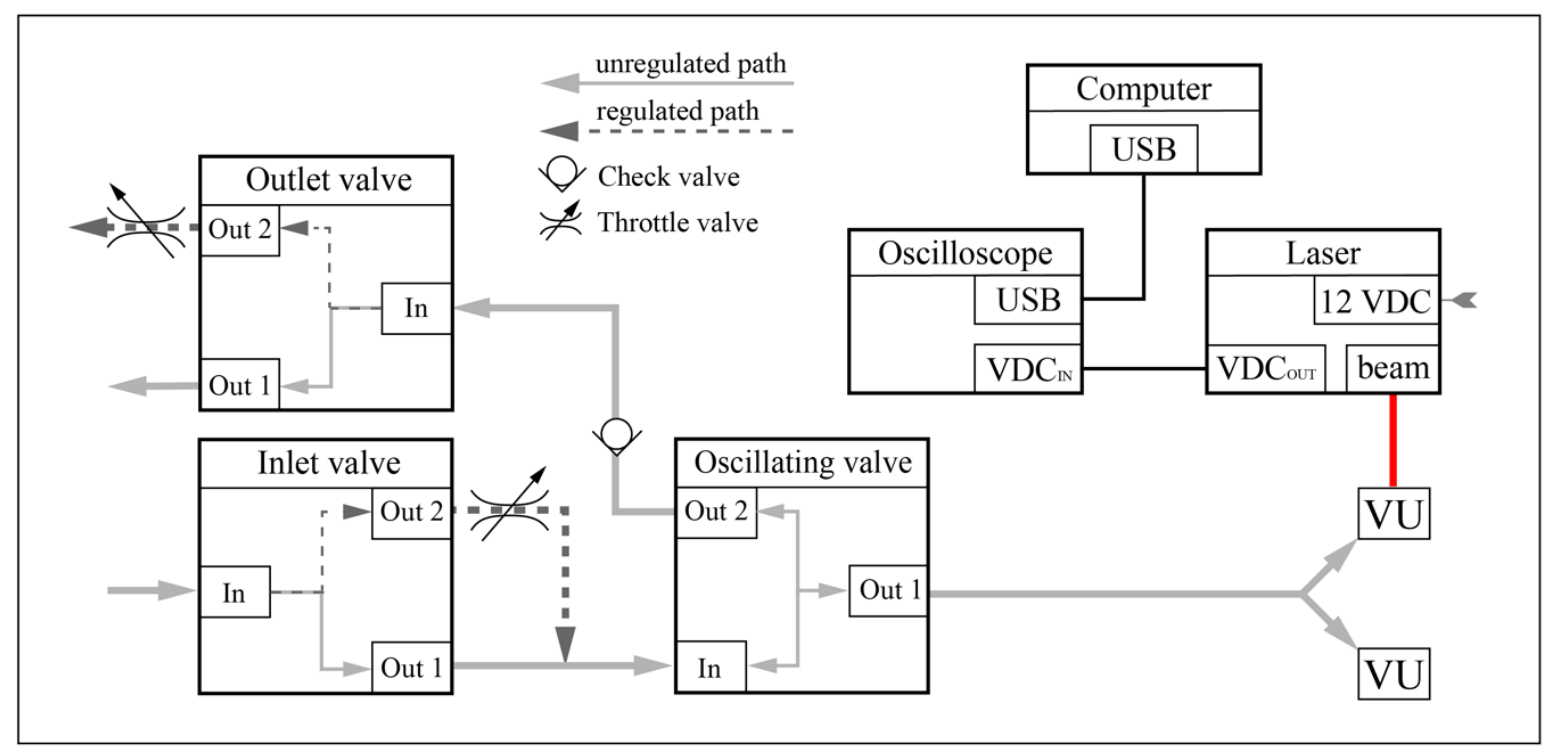

Figure 6: Schematic layout of the measuring system for measuring the VU's stimulation behaviour. The pneumatic set-up was the same as in section 2.4.1, except that only 1 instead of 10 oscillating fast-switching valve was used. 


\subsection{Assessment of the relationship between amplitude and frequency}

To investigate the relationship between frequency and amplitude, frequencies from $5-150 \mathrm{~Hz}$ with an increment of $5 \mathrm{~Hz}$, duration of the stimulation of $5 \mathrm{~s}$ and inter-stimulation time of $5 \mathrm{~s}$ were measured using the triangulation laser. The system was configured to operate at $20 \mathrm{~Hz}$ (regulated) and $80 \mathrm{~Hz}$ (unregulated).

\subsection{Assessment of delay time between start signal and stimulation start}

The delay of the signal generated by the Raspberry Pi 3B+ until the deflection of the VU was measured using the oscilloscope OpenScope MZ. For this purpose, both the triangulation laser and the active GPIO pin of the Raspberry Pi, which outputs the signal for the oscillating valve, were connected to the oscilloscope. Both channels of the oscilloscope were sampled at $4000 \mathrm{~Hz}$. A threshold of $0.1 \mathrm{~mm}$ was defined for detecting the deflection of the VU.

\subsection{Assessment of ramp up time of stimulation}

The ramp up time of the VU was defined by the threshold of $63.2 \%$ of the median amplitude of the VU, which corresponds to a fraction of $\kappa^{N} \kappa € ́$. The median amplitude was determined by observing the last 3 seconds of the stimulation period. This definition prevented the impact of possible overshoot processes. Both the unregulated system and the regulated system were analysed. The ramp up time of 10 VUs at $20 \mathrm{~Hz}$ (regulated) and $80 \mathrm{~Hz}$ (unregulated) stimulation was tested. Each VU was measured 10 times.

\subsection{Stability assessment of the stimulation amplitude}

10 runs with a single $\mathrm{VU}$ and frequencies $20 \mathrm{~Hz}$ (regulated) and $80 \mathrm{~Hz}$ (unregulated) were performed to assess the stability of the amplitude using the triangulation laser.

\subsection{Assessment of amplitude stability between different VUs}

To determine potential differences in amplitudes between individual VUs, 10 different VUs were analysed. Amplitudes of each VU were measured at $20 \mathrm{~Hz}$ (regulated) and $80 \mathrm{~Hz}$ (unregulated) over 10 measurement runs using the triangulation laser. The throttle valves were adjusted so that the amplitude at $20 \mathrm{~Hz}$ and $80 \mathrm{~Hz}$ was 0.7 $\mathrm{mm}$.

\subsection{Long-term assessments of the VU amplitude}

The VUs were tested on two timepoints within one year to assess their long-term behaviour regarding potential changes in amplitude (Figure 12). For these tests, the triangulation laser's sampling rate was set to $2000 \mathrm{~Hz}$ whereas the sampling rate of the digital oscilloscope was set to $4000 \mathrm{~Hz}$. The compressed air supply was set to $1.5 \mathrm{bar}$ for all measurements by means of a manometer (LEO1, Keller AG, Winterthur, Switzerland). The measurements were carried out with $10 \mathrm{VUs}$ that had been exposed to 30 pilot study subjects (total stimulation time approx. $60 \mathrm{~min}$ per subject, supine position in the MR) within the tested one-year period (approved by the Ethics commission of the Canton Zurich (ID 2021-01331). During this pilot experiments, the VUs were operated at the two stimulation frequencies $20 \mathrm{~Hz}$ and $80 \mathrm{~Hz}$. The regulated path for the $20 \mathrm{~Hz}$ stimulation was set to the amplitude of the unregulated path at $80 \mathrm{~Hz}$. Each of the $10 \mathrm{VUs}$ was assigned an individual ID (1-10). The analysis of the measurement data was performed using scripts written in Python. The amplitude was calculated based on the maximum and minimum displacement of each vibration pulse using the triangulation laser. The first and last 5 pulses of each stimulation sequence were discarded from the analysis to exclude possible settling and decay scenarios from the evaluation.

\subsection{Assessment of the relationship between VU amplitude and pressure application}

In the study setting, the VUs are under the influence of the study subject's body weight. Estimating the payload mass in human subjects is difficult because of the difference in weight and its distribution in the body. Conservative estimates yield estimated payloads between 50 and $200 \mathrm{~g}$ on paraspinal areas of the VU placement ${ }^{8,9}$. To assess the influence of the study subject's own weight on the amplitude of the VU, masses up to $400 \mathrm{~g}$ were applied. In the study setting, the skin of the study subjects is elastically deformed by the VU, which was not considered in the 
present analysis. Non-elastic brass cylinders guided in a linear sliding bearing were used as masses. It can be assumed that the amplitude of the VU is less affected in humans in the study setting situation due to skin elasticity.

\section{Results}

\subsection{Relationship between amplitude and frequency}

The amplitude depends on the respective stimulation frequency. As the stimulation frequency increases, the amplitude decreases, independent of whether the system is regulated or not (Figure 6). A potential explanation of this observation is as follows: The volume flow is constant. The time $\breve{T}^{\underline{s}}{ }_{\ell \sim}$ to fill the chamber of the VU shortens with increasing frequency. Therefore, the volume of air flowing into the chamber decreases accordingly. Assuming a constant ground area of the chamber, this implies that the amplitude must decrease like $\stackrel{\bullet}{e r}$ as well. This assumption is confirmed by the data and the guiding fit line in Figure 6.

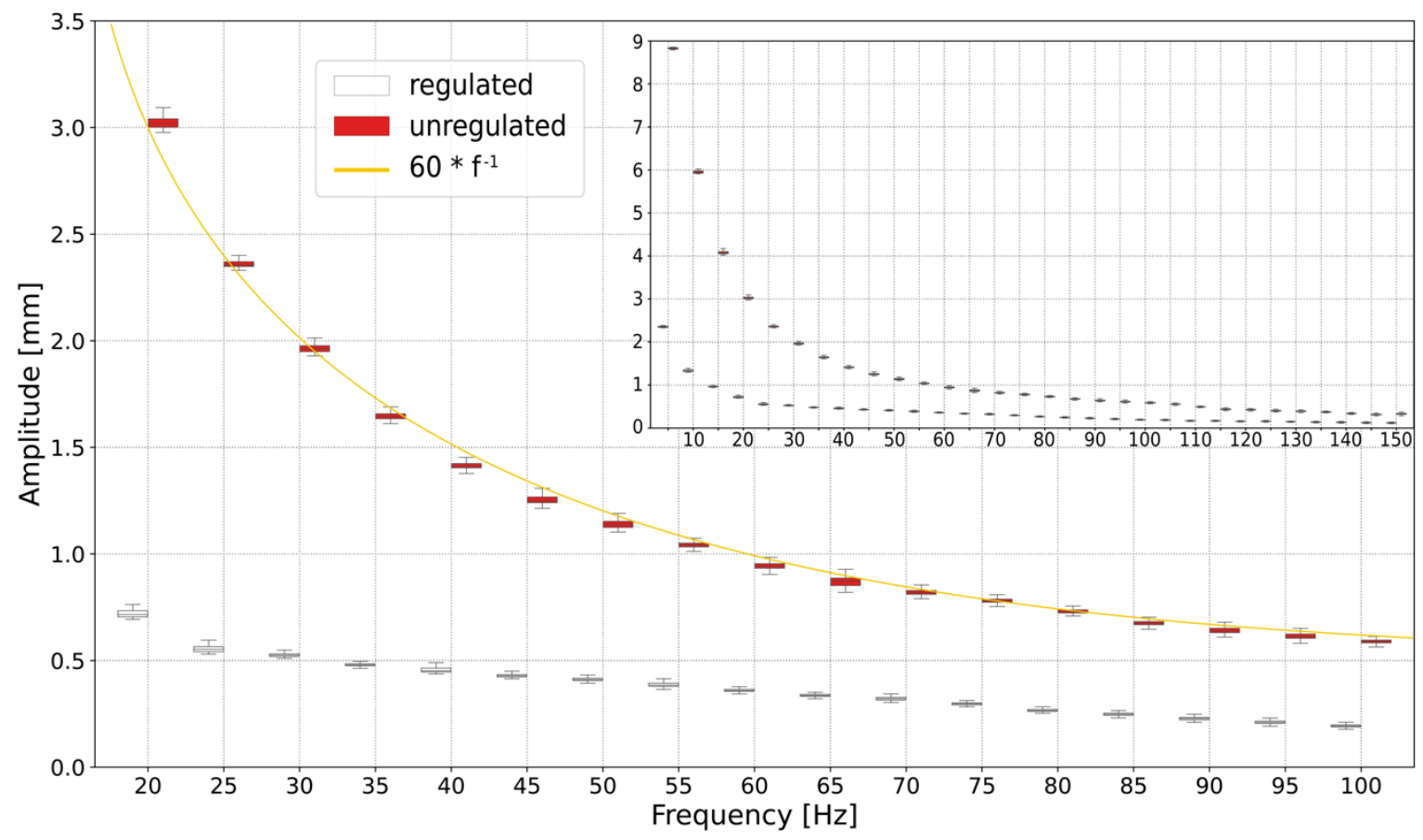

Figure 3: Relationship between the amplitude of the VU and the frequency, measured in each case with the regulated and the unregulated path. Measurements for frequencies from $5-150 \mathrm{~Hz}$ with an increase of $5 \mathrm{~Hz}$ are displayed in the inset. In the main plot, the relationship between frequency $(20-100 \mathrm{~Hz})$ and amplitude is shown. The yellow line represents the function $\mathrm{f}^{-1}$ with a multiplier of 60 . 


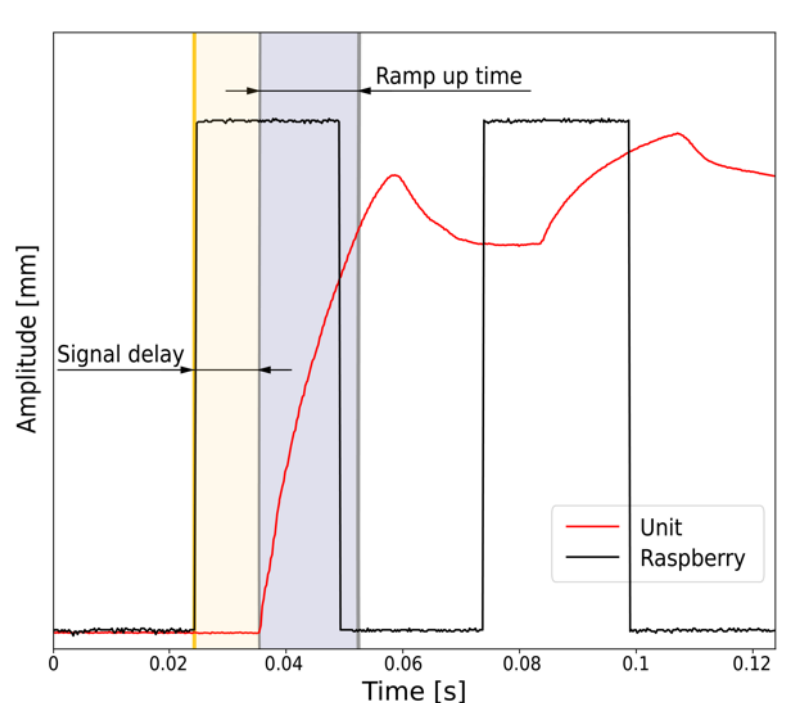

Figure 5: Signal delay, i.e. the time between the signal of the Raspberry Pi and the deflection of the VU. The signal delay is marked in yellow. The ramp up time is marked in grey (see section $3.3)$.
The signal delay refers to the time between the electronic impulse (raise of black line) and the start of the deflection of the VU (raise of red line) (Figure 7). The delay is primarily explained by the length of the tubing system and to a lesser extent by the activation time of the fastswitching valves $(<2 \mathrm{~ms})$. The ramp up time is defined as the time between the first movement of the VU (end of signal delay) and the time point where the VU reaches $63.2 \%$ of its final amplitude. All VUs demonstrated a very consistent distribution of the delay times. VU4 showed slightly higher values compared to the other VU. VU8 and 9 demonstrated slightly increased delay times for the $20 \mathrm{~Hz}$ stimulation frequency. The results of the ramp up time are displayed in the subsequence section (section 3.3).

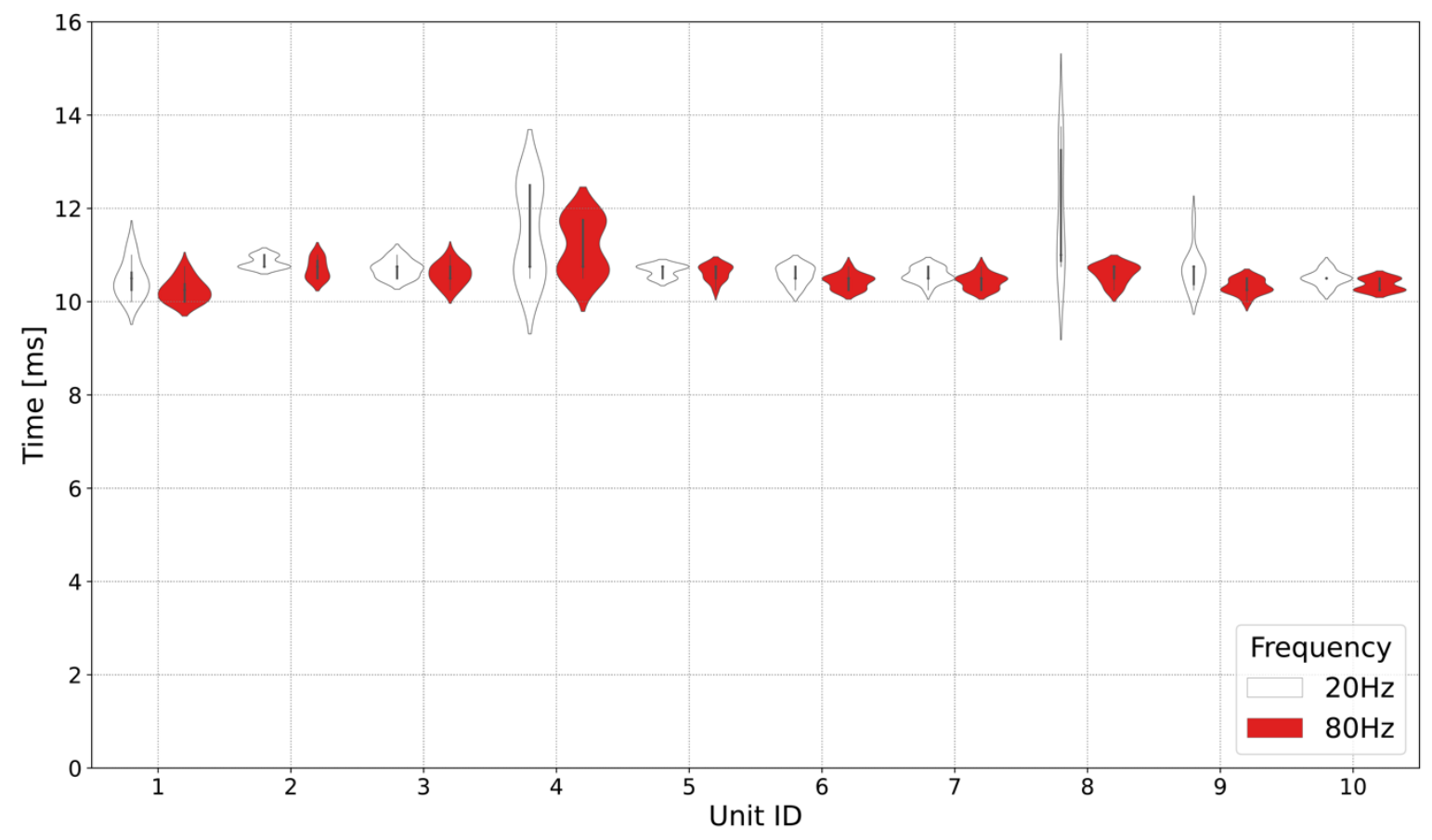

Figure 4: Violin plots showing the variability of the signal delay of 10 individual VUs based on 10 measurement runs per VU and frequency $(20 \mathrm{~Hz} / 80 \mathrm{~Hz})$. The signal delays ranged between $9.8-15.3 \mathrm{~ms}$. Unit no. 4 showed a stronger variation of the time needed for the deflection. This could be due to a defect or abrasion of the unit. Unit no. 8 showed a larger standard deviation of the signal delay for stimulation at $20 \mathrm{~Hz}$, which likely can be attributed to a measurement error, since the delay was not affected at $80 \mathrm{~Hz}$. 


\subsection{Ramp up time of stimulation}

The ramp up time for a stimulation with $20 \mathrm{~Hz}$ (respectively with the regulated path) is $10-15 \mathrm{~ms}$ longer compared to the $80 \mathrm{~Hz}$ (unregulated path) (Figure 9). The longer ramp up time of the deflection in the regulated system is to be expected, as the throttle check valves not only reduce the volume flow but also the flow velocity of the compressed air. As a consequence, the ramp up time is longer.

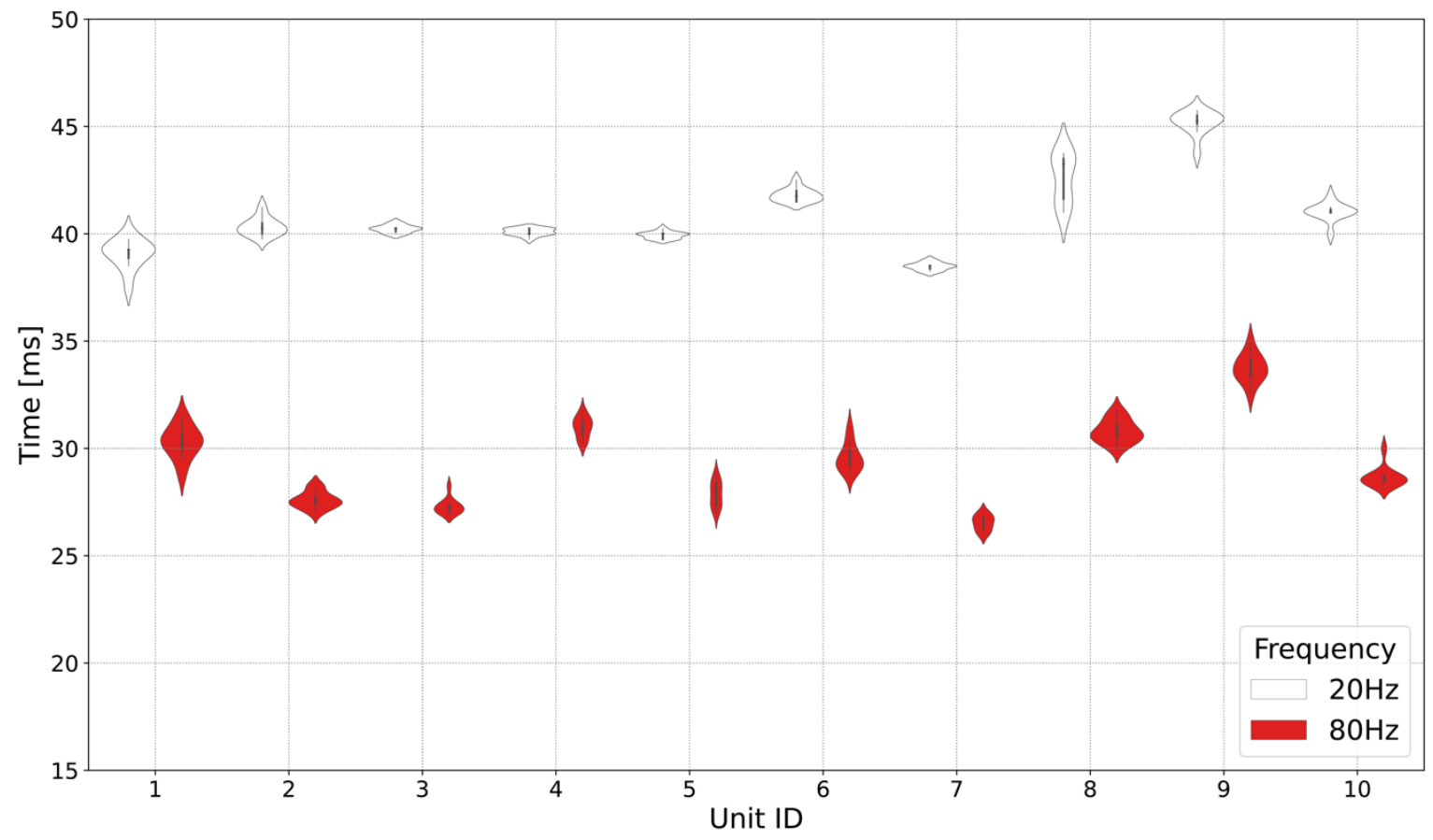

Figure 6: Violin plot showing the ramp up time of $10 \mathrm{VUs}$ based on 10 measurement runs for each frequency $(20 \mathrm{~Hz} / 80 \mathrm{~Hz})$. The difference between $20 \mathrm{~Hz}$ and $80 \mathrm{~Hz}$ is caused by the regulation of the volume flow during the $20 \mathrm{~Hz}$ stimulation (see section 3.1 ). 


\subsection{Amplitude stability}

The amplitudes were constant considering the 1-sigma intervals of variation of the amplitudes with small variation over all units regardless of the set frequency (Figure 10).

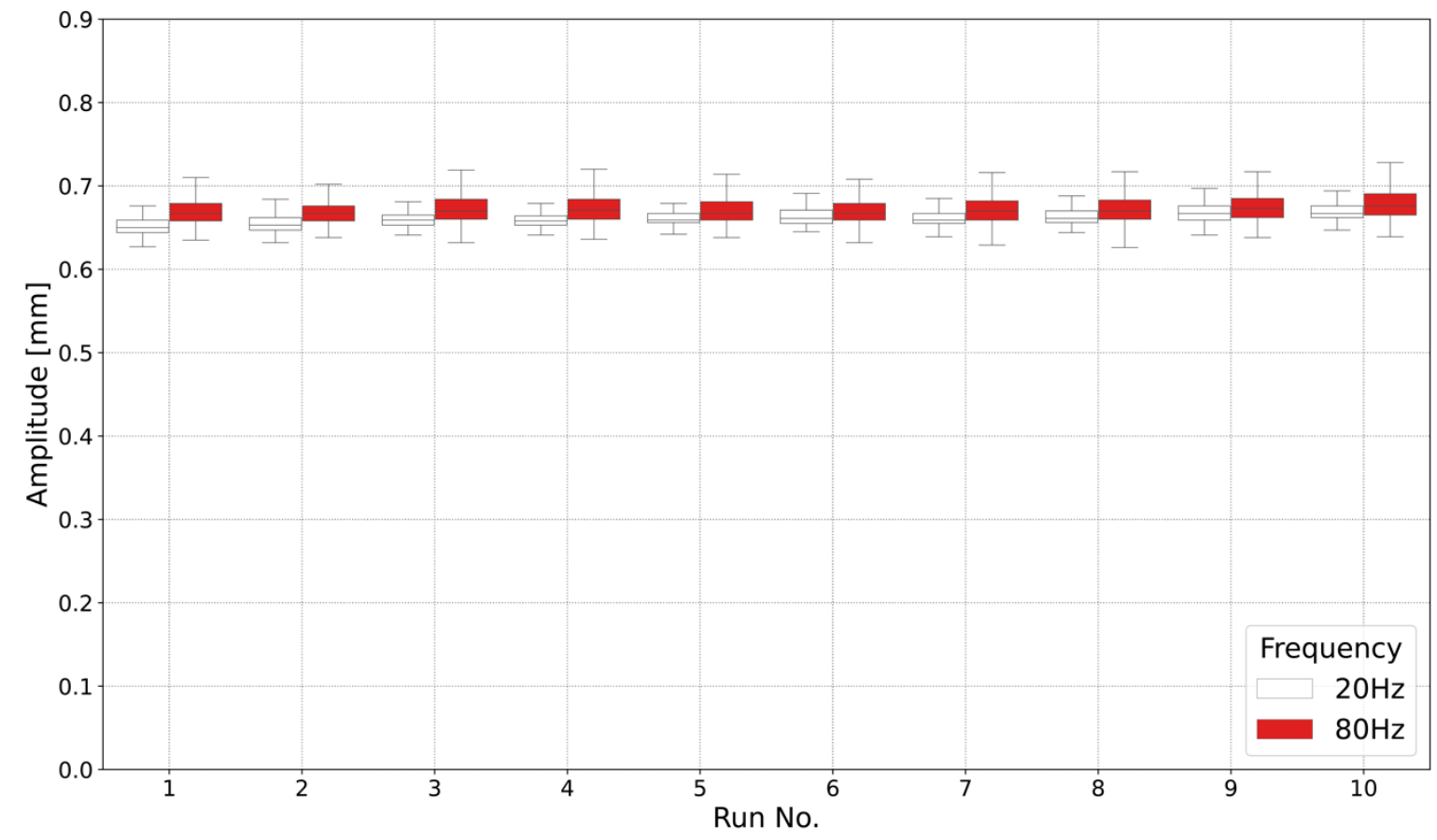

Figure 7: Boxplots showing the variability of amplitudes of $20 \mathrm{~Hz}$ and $80 \mathrm{~Hz}$ stimulation of Unit ID 1 based on 10 measurement runs per frequency $(20 \mathrm{~Hz} / 80 \mathrm{~Hz})$. 
The amplitudes between different VUs were in the desired range (0.5-1mm) (Figure 11).

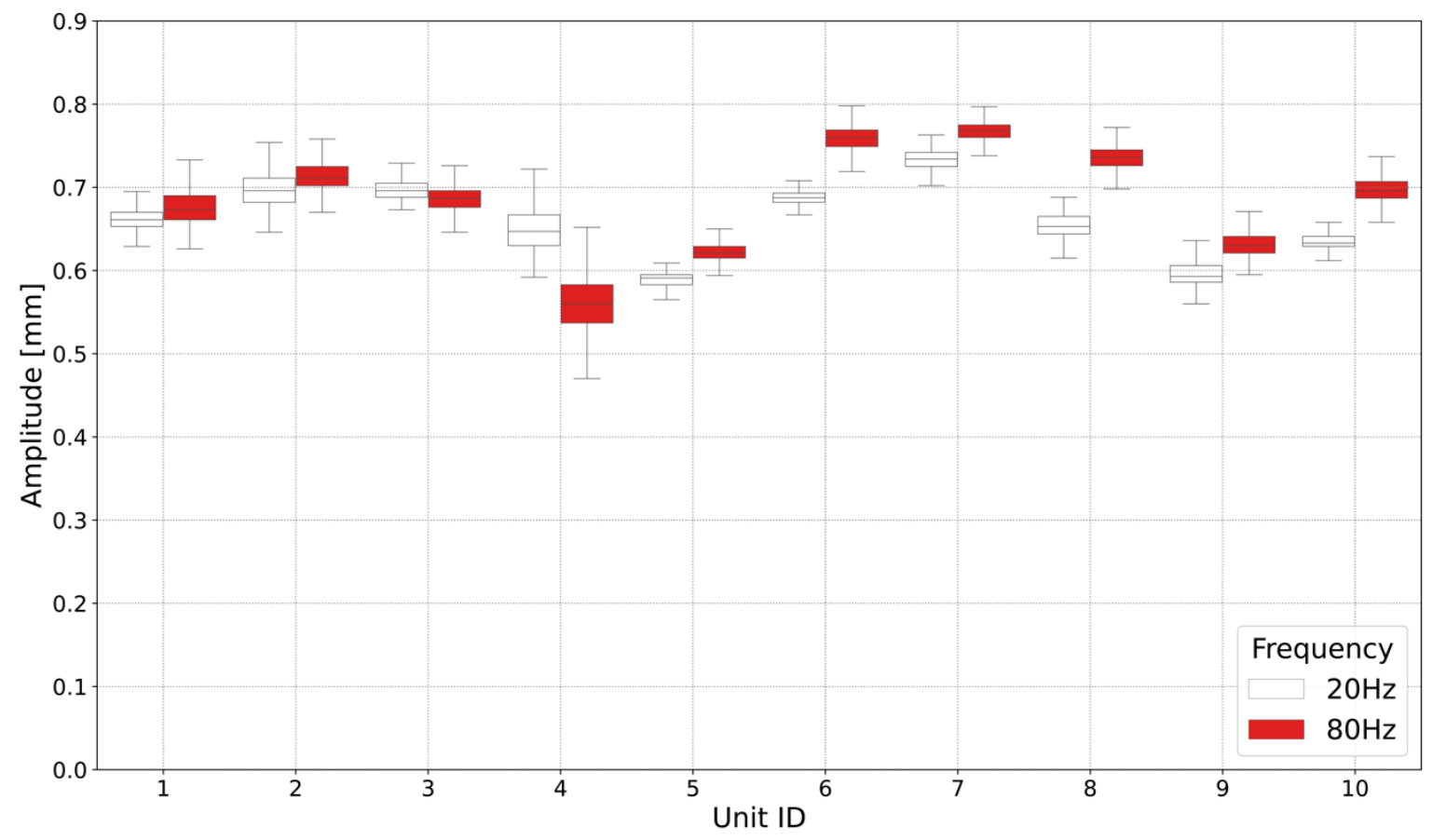

Figure 8: Boxplot showing the variability of the amplitudes of different VUs during $20 \mathrm{~Hz}$ and $80 \mathrm{~Hz}$ stimulation based on 10 measurement runs per VU and frequency $(20 \mathrm{~Hz} / 80 \mathrm{~Hz})$. 
The amplitudes of the VUs after one year of operation remained within the desired range (0.5-1mm) (Figure 12). A perfectly consistent VU would be represented on the diagonal (yellow line). Within one year, the mean stimulation amplitude dropped by only $13.3 \%$ on average at a frequency of $20 \mathrm{~Hz}$ and $2.9 \%$ at a frequency of $80 \mathrm{~Hz}$, respectively.

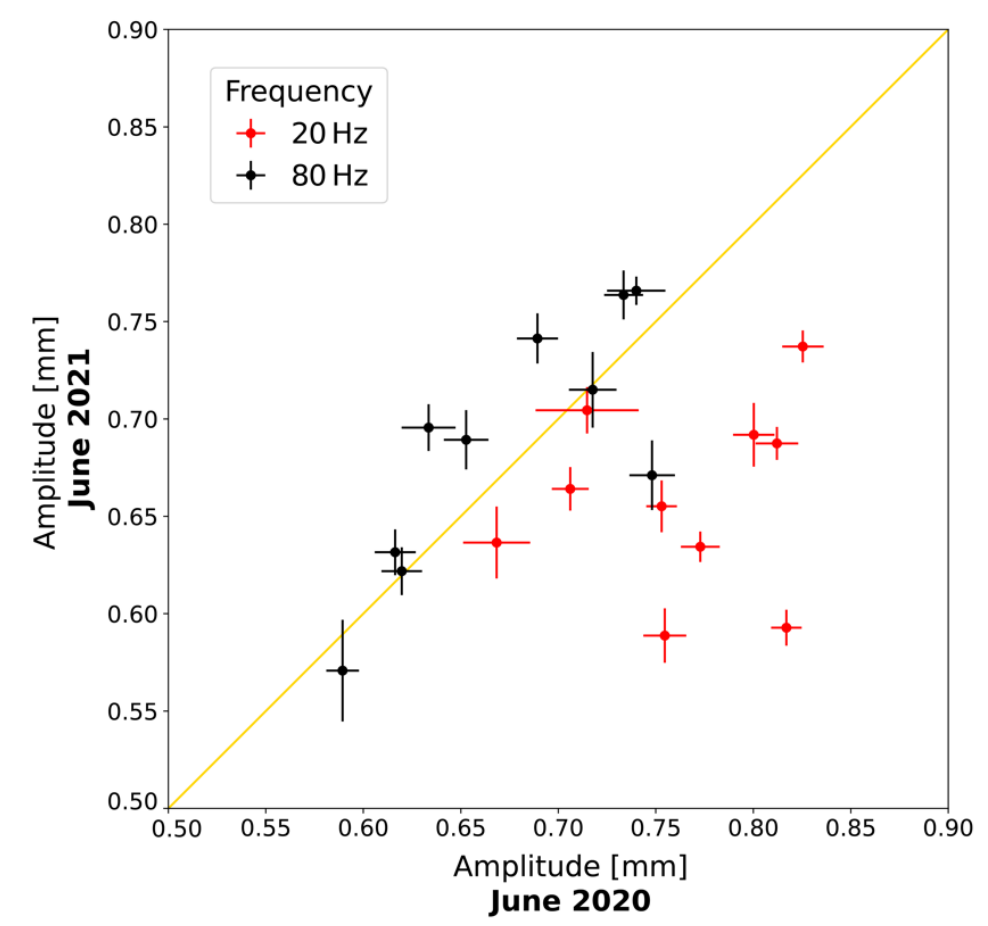

Figure 9: Change in amplitude of the VUs during $20 \mathrm{~Hz}$ and $80 \mathrm{~Hz}$ stimulation within one year of operation, shown as a scatterplot (based on 10 measurements runs per timepoint and frequency). 


\subsection{Relationship between VU amplitude and pressure application}

At an additional payload of $385 \mathrm{~g}$, the vibration amplitude displayed a clear break-down behaviour, i.e., the appropriate amplitude could not be delivered anymore (Figure 13). The addition of $383 \mathrm{~g}$ led to a reduction of the stimulation amplitude by $24.6 \%$ compared to the freely oscillating case. For masses between $100 \mathrm{~g}$ and $300 \mathrm{~g}$, the amplitude was in an acceptable range and was reduced by approx. $9 \%$ compared to the freely vibrating case.

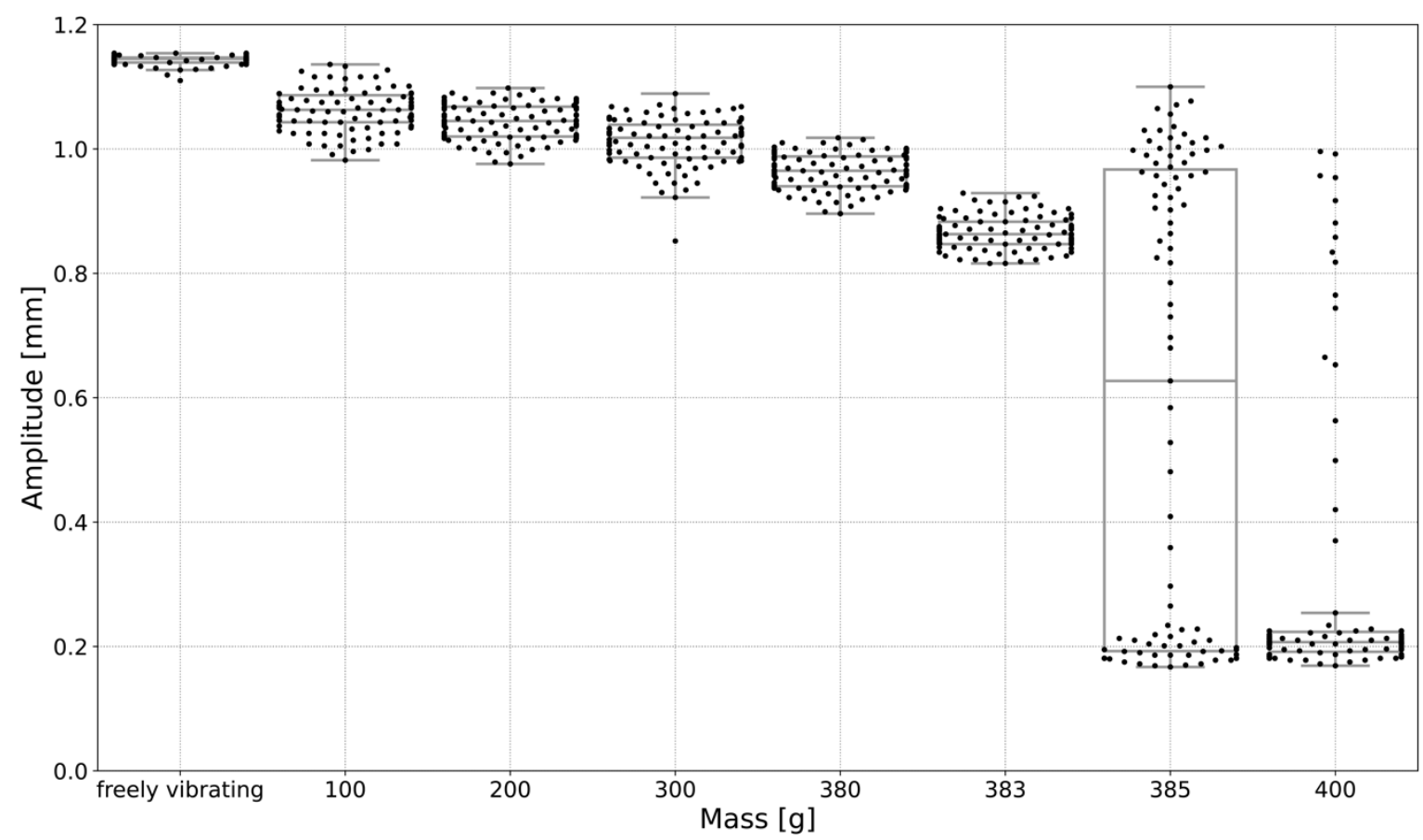

Figure 10: Swarm plots showing the influence of increasing payload mass on the amplitude of the VU $(20 \mathrm{~Hz})$ which helps in estimating the influence of body weight on the vibration amplitude in human study settings. A significant change in amplitude is visible above a mass load of $385 \mathrm{~g}$. 


\section{Conclusions}

PneuVID is the first apparatus specifically designed for human paraspinal tissue vibration in an MR environment. Vibrotactile stimulation is generated with manually produced VUs based on three layers of silicone and stabilisation grafts 3D printed in PLA. The excitation of the VUs is realized with pulses of compressed air operating at a feed pressure of 1.5 bar. The pulses of compressed air are generated by a valve box in the MR-measurement room operating fast-switching valves in combination with suitable driver electronics. The interaction with the MRsystem and the operator is provided by a control module connected via fibre optical connection to the valve box. PneuVID can deliver vibrotactile stimulation in a frequency range between $5-150 \mathrm{~Hz}$ including optimal stimulation parameters for muscle spindle activation $(80 \mathrm{~Hz}$ and amplitudes of $0.5-1 \mathrm{~mm})$. To guarantee consistent and identical stimulation amplitudes for the two investigated frequencies $(20 \mathrm{~Hz} / 80 \mathrm{~Hz})$, the system is equipped with passive pneumatic control devices that enable a regulation of the air flow and therefore an adjustment of the stimulation amplitudes. In summary, pneuVID represents a promising tool for assessing and differentiating the cortical sensory representation of paraspinal afferent inputs.

\section{Acknowledgements}

This work was supported by the Swiss National Science Foundation under grant 320030_185123. 


\section{References}

1. Buchbinder, R. et al. Low back pain: a call for action. Lancet (London, England) 391, 2384-2388; 10.1016/S0140-6736(18)30488-4 (2018).

2. Flor, H. Cortical reorganisation and chronic pain: implications for rehabilitation. Journal of rehabilitation medicine, 66-72; 10.1080/16501960310010179 (2003).

3. Sanchez-Panchuelo, R. M. et al. Within-digit functional parcellation of Brodmann areas of the human primary somatosensory cortex using functional magnetic resonance imaging at 7 tesla. The Journal of neuroscience : the official journal of the Society for Neuroscience 32, 15815-15822; 10.1523/JNEUROSCI.2501-12.2012 (2012).

4. Montant, M., Romaiguère, P. \& Roll, J.-P. A new vibrator to stimulate muscle proprioceptors in fMRI. Human brain mapping 30, 990-997; 10.1002/hbm.20568 (2009).

5. Carr, S. J., Borreggine, K., Heilman, J., Griswold, M. \& Walter, B. L. Novel magnetomechanical MR compatible vibrational device for producing kinesthetic illusion during fMRI. Medical physics 40, 112303 $10.1118 / 1.4824695$ (2013).

6. Proske, U. \& Gandevia, S. C. The proprioceptive senses: their roles in signaling body shape, body position and movement, and muscle force. Physiological reviews 92, 1651-1697; 10.1152/physrev.00048.2011 (2012).

7. Weerakkody, N. S., Mahns, D. A., Taylor, J. L. \& Gandevia, S. C. Impairment of human proprioception by high-frequency cutaneous vibration. The Journal of physiology 581, 971-980; 10.1113/jphysiol.2006.126854 (2007).

8. Hutchison, D. et al. (eds.). Advances in Visual Computing (Springer Berlin Heidelberg, Berlin, Heidelberg, 2012).

9. Stancic, I., Supuk, T. \& Cecic, M. Computer Vision System for Human Anthropometric Parameters Estimation. WTOS 8, 430-439 (2009). 